\title{
Multicenter Randomized Controlled Trial on the Provision of the EMDR-PRECI to Female Minors Victims of Sexual and/or Physical Violence and Related PTSD Diagnosis
}

\author{
Gabriela Jiménez ${ }^{1}$, Yael Becker ${ }^{2}$, Claudia Varela ${ }^{2}$, Paola García ${ }^{1}$, María Amparo Nuño ${ }^{1}$, \\ María Cristina Pérez ${ }^{1}$, Amalia Osorio ${ }^{1}$, Ignacio Jarero ${ }^{2, ~ *, ~ M a r t h a ~ G i v a u d a n ~}{ }^{2}$ \\ ${ }^{1}$ Department of Research, Ágape Desarrollo Humano Integral, Puebla City, Mexico \\ ${ }^{2}$ Department of Research, Mexican Association for Mental Health Support in Crisis, Mexico City, Mexico
}

\section{Email address:}

gabrielajimenezperegrina@gmail.com (G. Jiménez), yael406@gmail.com (Y. Becker), claudia_vc02@hotmail.com (C. Varela), paolagmyt@gmail.com (P. García), amparonuno@hotmail.com (M. A. Nuño), perezgrados@yahoo.com.mx (M. C. Pérez), amaliaosoriovigil@hotmail.com (A. Osorio),nacho@amamecrisis.com.mx (I. Jarero),martha@imifap.org.mx (M. Givaudan) ${ }^{*}$ Corresponding author

\section{To cite this article:}

Gabriela Jiménez, Yael Becker, Claudia Varela, Paola García, María Amparo Nuño, María Cristina Pérez, Amalia Osorio, Ignacio Jarero, Martha Givaudan. Multicenter Randomized Controlled Trial on the Provision of the EMDR-PRECI to Female Minors Victims of Sexual and/or Physical Violence and Related PTSD Diagnosis. American Journal of Applied Psychology. Vol. 9, No. 2, 2020, pp. 42-51. doi: 10.11648/j.ajap.20200902.12

Received: March 25, 2020; Accepted: April 8, 2020; Published: April 23, 2020

\begin{abstract}
This longitudinal multicenter randomized controlled trial has three objectives 1) to evaluate the EMDR-PRECI in the remission of the PTSD Diagnosis on female minors who were victims of sexual and/or physical violence compared to treatment as usual (TAU), 2) to evaluate the effectiveness of the EMDR-PRECI in the reduction of the PTSD, anxiety, and depression symptoms of the participants compared with TAU, and 3) to expand the knowledge on the existing correlation between the PCL-5 core criteria for PTSD provisional diagnosis and the CAPS-5 PTSD clinical diagnosis on this population. The study was conducted in 2019 in the cities of Puebla, Mexico, and Mexico City, in three sites of a non-profit organization who provide services to women in vulnerable situations. A total of 32 minors met the inclusion criteria. Participants' ages ranged from 12 to 17 years old ( $\mathrm{M}=15.35$ years old). Participation was voluntary with the participant's and their legal guardian's consent. It is relevant to mention that 31 of the 32 participants were pregnant during treatment with an average of 24.63 weeks (6.15 months) of pregnancy. All patients in the EMDR treatment group did not meet PTSD diagnostic criteria after treatment and at 90-days-follow-up assessments, while all patients in the TAU group maintained their PTSD baseline clinical diagnosis after treatment and at 90-days-follow-up assessments. Analyses of variance (ANOVA) for repeated measurements were used for PTSD, Anxiety and Depression; $t$ test and Cohen's $d$ effect size were calculated using within and between designs for the different measurements. ANOVA revealed a significant effect for time $\left(\mathrm{F}(2,52)=149.53 \mathrm{p}<.001, \eta_{P}{ }^{2}\right.$ $=.852)$, for group $\left(\mathrm{F}\left(1,26=140.71, \mathrm{p}<.001, \eta_{P}{ }^{2}=.844\right)\right.$ and Anxiety scores showed a significant effect for time $(\mathrm{F}(1,26)=$ $\left.32.89, \mathrm{p}<.001, \eta_{P}{ }^{2}=.559\right)$ and a significant interaction effect between time and group $\left(\mathrm{F}(1,26)=17.37, \mathrm{p}<.001, \eta_{P}{ }^{2}=.401\right)$ showed the decrease for the treatment group. For the depression scores significant effects were found for time $(\mathrm{F}(1,26)=$ 21.77, $\mathrm{p}<.001, \eta_{P}{ }^{2}=.456$. Results also showed significant interaction effects between time and group. $(\mathrm{F}(1,26)=6.17, \mathrm{p}<.05$, $\left.\eta_{P}{ }^{2}=.192\right)$. No significant effects for TAU group were founded. The study results indicate that the administration of the EMDR-PRECI could be a feasible, cost-effective, and time-efficient therapy to address PTSD diagnosis, and PTSD, anxiety and depression symptoms in this population.
\end{abstract}

Keywords: Eye Movement Desensitization and Reprocessing (EMDR), EMDR-PRECI, Posttraumatic Stress Disorder (PTSD), Female Minors, Sexual Violence, Physical Violence, Anxiety, Depression 


\section{Introduction}

In the context of this article, we will use the term "sexual violence" as an encompassing terminology of any behaviors related to sexual assault, aggression, harassment, abuse, rape, or grooming behaviors. According to the United Nations Children's Fund (UNICEF), child and adolescent sexual abuse are considered one of the most serious forms of abuse against persons under 18 years of age [1]. Its manifestations include different forms of sexual activity with minors ranging from sexual acts without physical contact such as exposure to pornographic material to others with body contact such as touching and penetration [2]. Sexually abused victims reported higher emotional reactions during and after trauma compared to victims of other trauma [3]. Posttraumatic Stress Disorder (PTSD) is one of the most common sequelae of child sexual abuse [4]. In school-age girls who were sexually abused, studies have shown the prevalence of $30 \%$ of dissociation symptoms and $40 \%$ of PTSD symptoms [5]. Child sexual abuse is also associated with severe negative short- and long-term mental health outcomes, such as depression, anxiety, and aggressive behavior [6]. A high prevalence of anxiety, depression, PTSD, and borderline personality disorder was found in women who experienced sexual abuse in childhood [7]. This data reveals that the consequences of this act can persist throughout adolescence and adulthood [8]. In Mexico, 20\% of women between the ages of 15 and 17 years old experienced some form of interpersonal violence in the family during 2015 [9]. According to studies by the Organization for Economic Cooperation and Development (OECD) published in 2019, among the countries belonging to this organization, Mexico ranks first in child sexual abuse with 5.4 million cases per year [10]. The children from particular ethnic groups could be more vulnerable to sexual symptoms of trauma. Besides socioeconomic status, family background, and abuse characteristics, it is also possible that cultural factors, which influence parenting, attitudes regarding sexuality, and symptom formation may help explain higher levels of sexual symptoms among different ethnic groups [11].

According to the Diagnostic and Statistical Manual of Mental Disorders ( $5^{\text {th }}$ edition; DSM-5), Posttraumatic Stress Disorder (PTSD) is a mental disorder occurring after exposure to one or more traumatic events (Criterion A) and is characterized by intense reliving of the traumatic event through intrusive memories and nightmares (criterion B); avoidance of reminders of the event (criterion $\mathrm{C}$ ); negative alterations in cognition and mood (criterion D); hypervigilance toward potential threats in the environment (criterion E); and in some cases, persistent or recurrent depersonalization symptoms. The Criteria A events included, but are not limited to, threatened or actual physical assault (e.g., childhood physical abuse, physical attack), threatened or actual sexual violence (e.g., forced sexual penetration, alcohol/drugfacilitated sexual penetration, abusive sexual contact, noncontact sexual abuse, sexual trafficking) (p.274) [12]

PTSD has multiple symptomatic phenotypes and is highly comorbid, especially among individuals who have experienced multiple traumas. The comorbidity of PTSD and depressive-anxiety disorders are sequelae of exposure to traumatic events [13]. Approximately half of the individuals with PTSD usually report comorbid depressive symptoms across various epidemiological samples [14]. PTSD can become a risk factor for other medical conditions, including some complications of pregnancy such as ectopic pregnancies, spontaneous abortions, hyperemesis, symptoms of premature delivery and excessive fetal growth [15]. It is likely and plausible that prenatal stress represents an important aspect of the intrauterine environment that would be expected to influence many, if not all, developmental outcomes [16]. Regarding the treatment of PTSD, recent meta-analyses and treatment guidelines recommend the application of Cognitive Behavior Therapy (CBT) or Eye Movement Desensitization and Reprocessing (EMDR) Therapy [17-20].

\subsection{EMDR Therapy}

Eye movement desensitization and reprocessing (EMDR) therapy is a structured eight-phase and three-pronged patientcentered psychotherapeutic comprehensive approach. EMDR therapy is guided by the adaptive information processing (AIP) theoretical model which posits that the primary source of psychopathology are memories of critical incidents or adverse life experiences that have been inadequately processed and maladaptively stored in a state-specific form [21]. These memories can be triggered by current internal and external stimuli, thus contributing to present dysfunction. EMDR therapy and trauma-focused Cognitive Behavioral Therapy (TF-CBT) are the only psychotherapies recommended for children, adolescents, and adults with PTSD in the WHO Guidelines for the Management of Conditions Specifically Related to Stress [22]. Recent research indicates that EMDR and Cognitive Behavioral Therapy (CBT) are effective treatment choices for both PTSD and Depressive Symptoms [23]. EMDR therapy has been reported to be effective with children suffering from trauma [24-25]. To the best of our knowledge, this is the first EMDR therapy study conducted in Latin America with LatinAmerican female minors who are victims of sexual and/or physical violence and related PTSD diagnosis.

\subsection{EMDR-Protocol for Recent Critical Incidents and Ongoing Traumatic Stress}

The EMDR Protocol for Recent Critical Incidents and Ongoing Traumatic Stress (EMDR-PRECI) is an eight-phase and three-pronged protocol developed in the field specially designed to treat recent, present or past prolonged adverse experiences (e.g., sexual and/or physical violence, interpersonal violence, ongoing or prolonged traumatic stress). EMDR-PRECI conceptualizes the continuum of prolonged adverse experiences as creating a cumulative trauma exposure memory network of linked pathogenic memories with similar emotional, somatic, sensorial and 
cognitive information that does not give the cumulative statedependent traumatic memory network sufficient time to consolidate into an integrated whole [26]. The EMDRPRECI was selected to treat this population because patients were living with ongoing traumatic stress due to their vulnerable situation and also presented a cumulative trauma exposure memory network of linked sexual and/or physical violence-related pathogenic memories. For more information about this protocol, see Jarero, Artigas, and Luber [27].

\subsection{Previous EMDR-PRECI Studies}

The EMDR-PRECI has seven previous studies that have proved its efficacy with different populations: (i) with survivors of a 7.2 earthquake, (ii) with forensic personnel after a human massacre, (iii) with traumatized first responders, (iv) with technological disaster survivors, (v) with family caregivers of patients with an autism spectrum disorder, (vi) with first responders on active duty, and (vii) with female patients with cancer-related PTSD diagnosis [27-34].

\section{Objectives}

This Longitudinal Multicenter Randomized Controlled Trial (RCT) has three objectives.

1. To evaluate the effectiveness of the EMDR-PRECI in the remission of the Posttraumatic Stress Disorder (PTSD) diagnosis on female minors who were victims of sexual and/or physical violence and related PTSD diagnosis compared to treatment as usual (TAU; psychological support, oriented to life plan and emotions management).

2. To evaluate the effectiveness of the EMDR-PRECI in the reduction of the PTSD, anxiety, and depression symptoms of the participants compared to TAU.

3. To expand the knowledge on the existing correlation between the Posttraumatic Stress Disorder Checklist for DSM-5 (PCL-5) core criteria for PTSD provisional diagnosis and the Clinician-Administered PTSD Scale for DSM-5 (CAPS-5) PTSD clinical diagnosis on this population.

\section{Method}

\subsection{Study Design}

The current study is a two parallel armslongitudinal randomized controlled trial (RCT). This research design allowed us to determine the effectiveness of the EMDRPRECI in PTSD diagnosis remission and PTSD, anxiety and depression symptom reduction compared to treatment as usual (TAU).

\subsection{Ethics and Research Quality}

The research protocol was reviewed and approved by Vida y Familia (Life and Family; VIFAC) Institutional Review Board (IRB) in compliance with the International Committee of Medical Journal Editors recommendations, the Guidelines for Good Clinical Practice of the European Medicines Agency (version 1 December 2016) and the Helsinki Declaration as revised in 2013. The research quality of this study was based on the Consolidated Standards of Reporting Trials (CONSORT) 2010 Statement and the Standard Protocol Items Recommendation for Interventional Trials (SPIRIT) 2013 checklist [35-36].

\subsection{Participants}

This study was conducted in 2019 in the cities of Puebla, Mexico and Mexico City, Mexico in three of the VIFAC centers. VIFAC is a non-profit agency founded in 1985 who provide services to women in vulnerable situations (e.g., without family support, job, savings) and in many cases pregnant. The enrollment was consecutive. Forty-six potential participants were recruited at the three VIFAC centers (one in Puebla and two in Mexico City). Fourteen were excluded (12 on the basis of the inclusion/exclusion criteria and 2 declined to participate in the study). A total of 32 victims of sexual and/or physical violence fulfilled the inclusion criteria and were enrolled in the study. Participants' age ranged from 12 to 17 years old $(\mathrm{M}=15.35)$. Participation was voluntary, with the consent of both the participants and their legal guardians. It is relevant to mention that 31 of the 32 participants were pregnant during treatment with an average of 24.63 weeks ( 6.15 months) of pregnancy.

Inclusion criteria were: (a) being a minor victim of sexual and/or physical violence living in one of the VIFAC centers, (b) having met the DSM-5 Criteria A, (c) having a current PTSD diagnosis of sexual and/or physical violence established by the Clinician-Administered PTSD Scale for DSM-5 (CAPS-5), (d) voluntarily participating in the study, (e) not receiving current specialized trauma therapy, (f) not receiving drug therapy for posttraumatic stress disorder symptoms.

Exclusion criteria were: (a) ongoing self-harm/suicidal or homicidal ideation, (b) active diagnosis of schizophrenia, psychotic or bipolar disorder, (c) diagnosis of dissociative disorder, (d) organic mental disorder, (e) a current, active chemical dependency problem, (f) significant cognitive impairment (e.g., severe intellectual disability, dementia), g) presence of uncontrolled symptoms due to medical illness.

\subsection{Randomization, Allocation Concealment Mechanism, and Blinding Procedure}

A simple randomization with a 1:1 allocation ratio was used. Two independent assessors blind to treatment conditions conducted the randomization process to avoid allocation influence. The treatment allocation sequence was concealed using sequentially numbered, opaque, sealed and stapled envelopes. The safekeeping of the envelopes and the assignment of participants to each arm of the trial was overseen by a person not involved in the research study.

The treatment allocation of the participants was blinded for the mental health professionals who conducted the intake interview, the research assistants who conducted the initial 
assessment and enrollment and for the independent assessors who conducted the follow-up assessments. Participants were instructed to not reveal their treatment allocation to the persons conducting the assessments. Based on ethical considerations, participants in the TAU condition were offered EMDR-PRECI after the follow-up assessment. Eighteen participants were allocated in the treatment group and eighteen participants in the control group. See Flow Diagram in Figure 1.

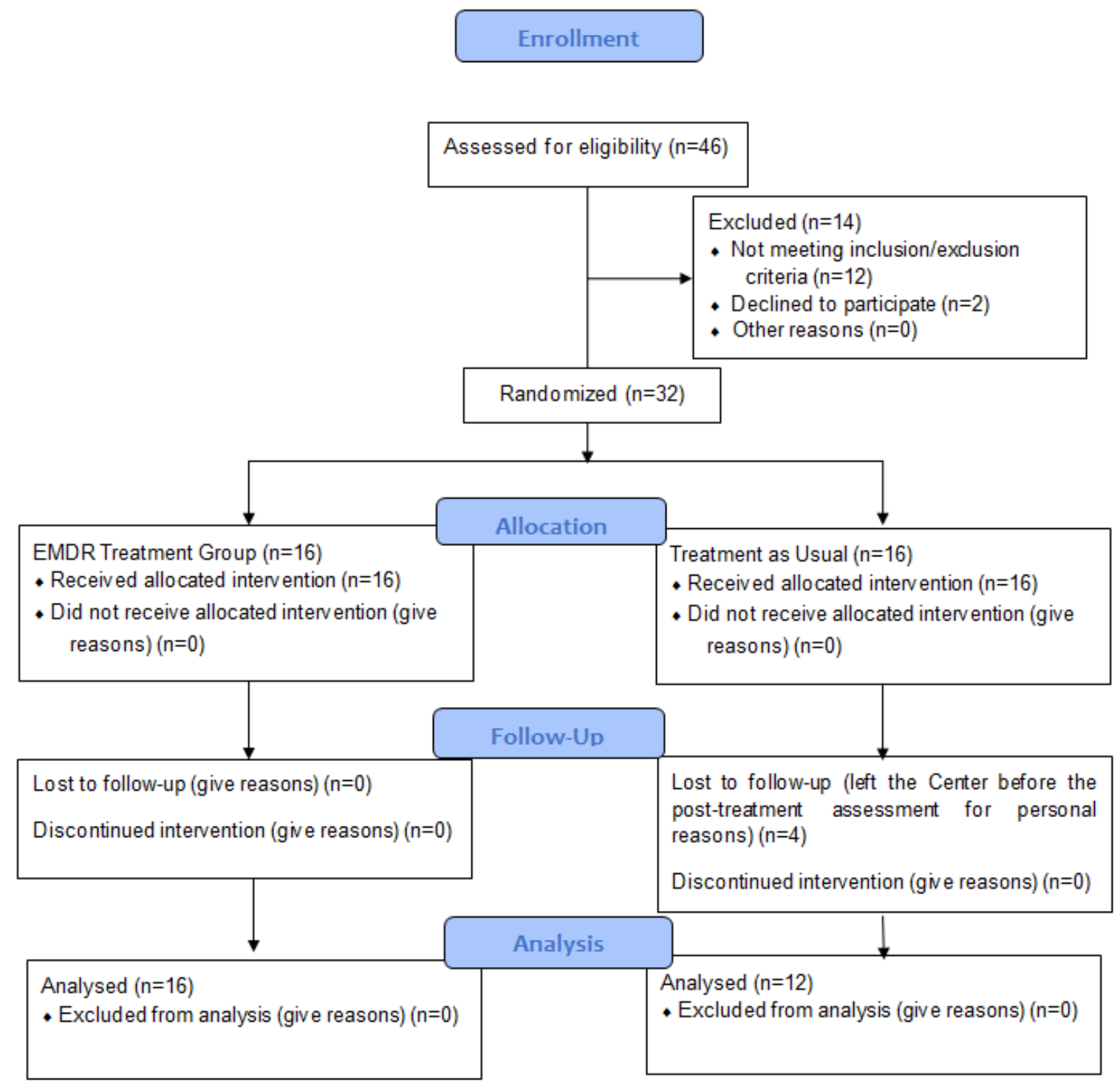

Figure 1. Flow Diagram.

\subsection{Instruments}

1) The Clinician-Administered PTSD Scale for the DSM5 (CAPS-5) is a structured diagnosis interview and is the gold standard for assessing PTSD [37]. A 5-point ordinal rating scale is used to measure symptom severity (ranging from $0=$ absent to 4 = extreme extreme/incapacitating) on the 20 symptoms that correspond to PTSD diagnosis. Symptoms severity ratings combine information about symptoms frequency and intensity obtained by the interviewer. The identified symptoms qualify as criterion meeting a PTSD diagnosis as established by the DSM-5. The questions review onset and duration of symptoms, subjective distress, impact of symptoms on occupational and social functioning, overall response validity, and severity of symptoms. Scoring is calculated by summing the total score for severity and additionally categorizing each of the 20 questions into the appropriate symptom cluster by calculating the individual severity scores for symptoms corresponding to a given DSM-5 cluster. Besides the symptoms that correspond to PTSD diagnosis, this instrument has Global Ratings on Global Validity (item 26), Global Severity (item 27), and Global Improvement (item 28). This last item has six rating options: 0) Asymptomatic, 1) Considerable Improvement, 2) Moderate Improvement, 3) Slight Improvement, 4) No Improvement, and 5) Insufficient Information. Lastly, items 29 and 30 are about depersonalization and derealization symptoms respectively. The assessment is administered by a trained person in order 
to assure accuracy in understanding and consistency. In the current study, PTSD diagnosis status was established according to the DSM-5 diagnostic algorithm and using the CAPS-5 Child/Adolescent Version. The instrument was translated and back-translated to Spanish.

2) We used the Posttraumatic Stress Disorder Checklist for DSM-5 (PCL-5) to expand the knowledge on the existing correlation between this instrument core criterion for PTSD provisional diagnosis and the Clinician-Administered PTSD Scale for DSM-5 (CAPS-5) PTSD clinical diagnosis on this population [38-39]. The instrument was translated and backtranslated to Spanish. It contains 20 items, including three new PTSD symptoms (compared with the PTSD Checklist for DSM-IV) [40]: blame, negative emotions, and reckless or self-destructive behavior. Respondents indicate how much they have been bothered by each PTSD symptoms over the past month, using a 5 -point scale ranging from $0=$ not at all, $1=$ a little bit, $2=$ moderately, $3=$ quite a bit and $4=$ extremely. A total-symptoms score of zero to 80 can be obtained by summing the items. The sum of the scores yields a continuous measure of PTSD symptom severity for symptom clusters and the whole disorder. Psychometrics for the PCL-5, validated against the Clinician-Administered PTSD Scale-5 (CAPS-5) diagnosis, suggest that a score of 31-33 is optimal to determine probable PTSD diagnosis [41], and a score of 33 is recommended for use at present.

The PCL-5 is intended for a variety of clinical and research assessment tasks, including quantifying PTSD symptom severity, measuring the underlying construct of PTSD, establishing a provisional PTSD diagnosis, and estimating the presumed prevalence of PTSD. A score decrease between five to ten points demonstrates a clinically significant change. Decision rules for a provisional PTSD diagnosis based on PCL-5 core criteria are very accurate compared to CAPS diagnosis results. It is important to mention that at the first assessment meeting, before answering the CAPS-5 and the PCL-5, all participants were asked to focus specifically on the worst sexual and/or physical violence-related event that currently bothered them the most; then at each subsequent assessment meeting, they were asked to focus on the same event.

3) The Hospital Anxiety and Depression Scale (HADS) has been extensively used to evaluate these psychiatric comorbidities in various clinical settings at all levels of healthcare services and with the general population [42-43]. The instrument was translated and back-translated to Spanish. It is a 14 item self-report scale to measure the anxiety (7 items) and depression ( 7 items) of patients with both somatic and mental problems using a 4-point Likert scale ranging from 0 to 3. The response descriptors of all items are Yes, definitely (score 3); Yes, sometimes (score 2); No, not much (score 1); No, not at all (score 0). A higher score represents higher levels of anxiety and depression: a domain score of 11 or greater indicates anxiety or depression; 8-10 indicates borderline case; 7 or lower indicates no signs of anxiety or depression.

\subsection{Procedure}

\subsubsection{Assessments Times, Enrollment, Blind Data Collection, and Confidentiality of Data}

Treatment and control group participants completed the instruments on an individual basis in the different measurement moments. During time 1, mental health professionals working in each center conducted the intake interview and collected clinical histories. After this procedure, research assistants formally trained in all the instrument administration who were not blind to the study but blind to the treatment allocation assessed potential participants for eligibility based in the inclusion/exclusion criteria, obtained signed informed consent from the participants and their legal guardians, conducted the pre-treatment application of instruments, and enrolled participants in the study. During Time 2 (post-treatment assessment 30 days after treatment) and Time 3 (follow-up assessment 90 days after treatment) assessment was conducted for all participants by blind to treatment allocation independent assessors with a master's degree in clinical psychology and formal training in all the instrument administration. All data was collected, stored, and handled in full compliance with the VIFAC requirements to ensure confidentiality. The legal guardians of each study participant gave their consent for access to their personal data, which was strictly required for study quality control. All persons involved in this research project were subject to professional confidentiality.

\subsubsection{Withdrawal from the Study}

All research participants had the right to withdraw from the study without justification at any time and with assurances of no prejudicial result. If participants decided to withdraw from the study, they were no longer followed up in the research protocol. There were four withdrawals in the control group before Time 2 (post-treatment assessment) for personal reasons not related with the study. See Flow Diagram in Figure 1.

\subsubsection{Treatment}

In this study, intensive EMDR therapy was provided [4445]. Evidence suggests that more frequent scheduling of treatment sessions maximized PTSD treatment outcomes [46] This intensive format allowed the participants to complete the full course of treatment in a short period. Participants in the treatment group completed an average of 4.68 sixtyminutes individual sessions of EMDR treatment provided two or three times a week (depending on the availability of the participants) at the centers while the Treatment as Usual participants received an average of 12.6 sixty-minute individual sessions provided once a week.

Therapists and Treatment Fidelity

EMDR-PRECI was provided by eight licensed EMDR clinicians formally trained in the protocol administration. To protect the identity of the minors, no videotape or photographs were allowed. Treatment fidelity and adherence to the protocol were fulfilled by EMDR therapists' strict observance of all steps of the 
scripted protocol.

EMDR-PRECI Treatment Description and Tolerance.

Each EMDR-PRECI session lasted 60 minutes. The minimum number of sessions was two and the maximum was nine with an average of 4.68. Treatment focused only on the distressing memories related to the sexual and/or physical violence experiences and did not address any other memories To encompass the whole violence-related cumulative trauma exposure memory network, clinicians asked each of the participants to remember the worst sexual and/or violencerelated memory that they selected during the pretreatment CAPS-5 and PCL-5 assessments. Once the clients had reprocessed the worst memory, clinicians asked them to run a mental movie of the whole sexual and/or physical violencerelated memory network from right before the beginning until today, or even looking into the future (to reprocess flash-forwards when these are present), and then to identify any other disturbing parts/memories [47-48]. When the entire sexual and/or physical violence experience was visualized from start to finish (including flash-forwards) without emotional, cognitive, or somatic distress; the clinician elicited the representative Positive Cognition (PC) for the entire experience. Once the client visualized the entire experience, feeling the PC was completely true and without emotional, cognitive, or somatic distress, the clinician conducted the Body Scan Phase in which the client reprocessed any disturbance and/or strengthened positive affect or body sensations. Following the EMDR-PRECI's three-pronged approach, once the past memories had been reprocessed, the clinician looked for present triggers and conducted a future template. All participants reprocessed more than one pathogenic memory. No adverse effects or pregnancy complications were reported during EMDR treatment or at a three-month post-treatment assessment.

Examples of Worst Experiences Reprocessed During Treatment

Examples of the study participants' worst experiences: (1) A 17 year-old participant that was raped at six years old by her two brothers and had been repeatedly brutally beaten by her father had, on one occasion, spent up to a week in bed recovering. (2) A 15 year-old participant raped at 12 years old by a neighbor's son. After telling her mother what happened, the mother tried to strangle her because "surely you provoked him." A few months later the aggressor was killed with multiple stabs in the face and the whole body. The mother forced her to see the body, an image she could not erase. (3) A 12 year-old participant was sexually abused for five hours by her mother's boyfriend and she was afraid to tell her about it. A few months later when the mother found out about her daughter's pregnancy, she accused her daughter of seducing the boyfriend and expelled her from the house. (4) A 13-year-old participant was sexually abused by her uncle, her brother and the next-door neighbor through economic compensation and her mother's consent. The participant is illiterate, doesn't know the difference between right and wrong, described feeling a profound sadness and showed an aggressive behavior. (5) A 17 year- old participant was forced to live with her parents during her pregnancy because her husband was working for a drug cartel. While she was staying with them her alcoholic father and brother got into a fight with her younger brother and they murdered him in front of her. (6) A 15 year-old participant, originally from El Salvador, arrived in Mexico City after being sexually abused and threatened by three drug addicts. She fled her country with her 2-year-old daughter leaving her husband and family behind. She constantly heard in her head her attacker's laughter and couldn't sleep; she was constantly tormented by the whole experience. (7) A 17-year-old participant was brutally abused by her partner, to the point where she miscarried two babies. Humiliated through verbal abuse, she was made to sleep on the cold floor and thrown out of her own house in the middle of the night, with nowhere to go, while pregnant with her child. (8) A 14 year-old participant was sexually abused as a child by her mother's boyfriend more than once. When she finally told her mother what had happened, her mother didn't believe her. Her mother expelled her from the house and kept the relationship with the boyfriend.

\section{PTSD Diagnosis Status and Statistical Analysis.}

PTSD diagnosis status and statistical analysis was conducted for those participants, in both treatment $(\mathrm{N}=16)$ and TAU $(\mathrm{N}=12)$ groups, who fulfilled the inclusion criteria of PTSD at the beginning of the study and completed the post-treatment and the follow-up assessments. Analyses of variance (ANOVA) for repeated measurements were used for PTSD, Anxiety and Depression symptoms; $t$ test and Cohen's $d$ effect size were calculated using within and between designs for the different mean comparisons.

\section{Results}

\section{PTSD Diagnosis Remission}

PTSD diagnosis status, established according the DSM-5 diagnostic algorithm, showed that participants in the EMDR group (16 out of $16,100 \%)$ did not meet PTSD diagnostic criteria after treatment and at 90-days follow-up assessments, while all patients in the TAU group maintained their PTSD baseline clinical diagnosis after treatment and at 90-daysfollow-up assessments. In reference to the CAPS-5 Global Improvement (item 28), EMDR treatment group participants showed the following results: 8 out of $16(50 \%)$ reported being asymptomatic, and the other $8(50 \%)$ reported a considerable improvement.

\section{PTSD Symptoms}

Data analysis by repeated measures ANOVA revealed a significant effect for time $\left(\mathrm{F}(2,52)=149.53 \mathrm{p}<.001, \eta_{P}^{2}\right.$ $=.852)$, a significant effect for group $(\mathrm{F}(1,26=140.71$, $\left.\mathrm{p}<.001, \eta_{P}{ }^{2}=.844\right)$ and a significant interaction between time and group, $\left(\mathrm{F}(2,52)=146.75, \mathrm{p}<.001, \eta_{P}^{2}=.849\right)$. 
Comparison between groups did not show significant differences for time 1. For time 2 significant differences among the groups were found, $\mathrm{t}(26)=-11.14, \mathrm{p}<.001, d=$ 4.25 and for time 3 significant differences between groups were confirmed, t $(26)=-20.69, \mathrm{p}<.001, d=7.90$. In the treatment group, mean scores showed a significant decrease between time 1 and time $2, \mathrm{t}(15)=14.05, \mathrm{p}<.001, d=3.66$. There was also a significant decrease between time 2 to time $3, \mathrm{t}(15)=2.02, \mathrm{p}<.01, d=.38$, and between time 1 and time 3 , $\mathrm{t}(15)=23.39, \mathrm{p}<.001, d=-4.25$. In the TAU group no significant differences were found between means in all time comparisons. See Table 1 and figure 2 .

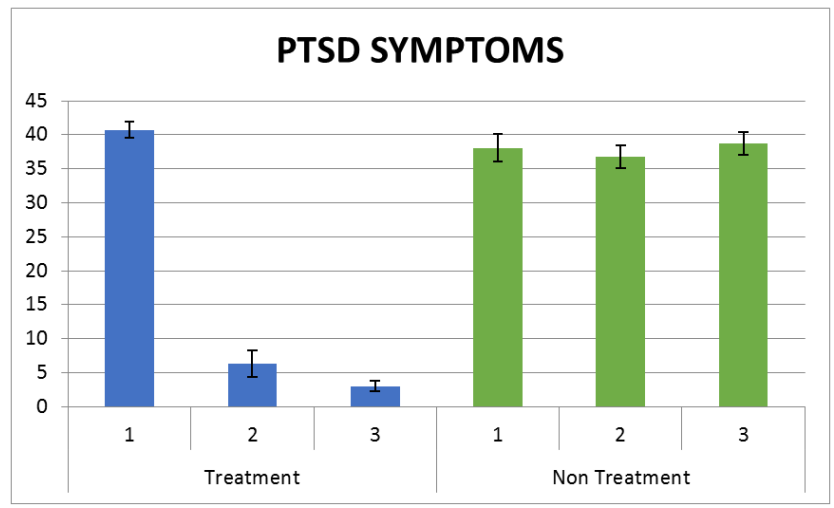

Figure 2. Mean scores and standard error for PTSD symptoms by time and group.

Table 1. Mean scores $(M)$ and standard deviations (SD) for treatment and no-treatment group on pre-test, post-test and follow up measurements.

\begin{tabular}{lllllll}
\hline & Time 1 & \multicolumn{3}{c}{ Time 2 } & \multicolumn{3}{c}{ Time 3 } \\
\cline { 2 - 7 } & M & SD & M & SD & M & SD \\
\hline PSTD & & & & & & \\
Treatment group & 40.75 & 4.89 & 6.31 & 8.02 & 3.00 & 3.16 \\
No treatment group & 38.08 & 6.97 & 36.83 & 5.81 & 38.75 & 5.89 \\
Anxiety & & & & & & \\
Treatment group & 10.62 & 4.48 & & & 3.25 & 2.64 \\
No treatment group & 9.08 & 4.87 & & & 7.91 & 4.12 \\
Depression & & & & & & \\
Treatment group & 8.18 & 4.32 & & & 3.00 & 2.65 \\
No treatment group & 5.58 & 4.12 & & & 4.00 & 2.82 \\
\hline
\end{tabular}

Anxiety

Repeated measures analysis of variance (ANOVA) for anxiety showed a significant effect for time $(F(1,26)=$ $\left.32.89, \mathrm{p}<.001, \eta_{P}{ }^{2}=.559\right)$ and a significant interaction effect between time and group $(\mathrm{F}(1,26)=17.37, \mathrm{p}<.001$, $\left.\eta_{P}{ }^{2}=.401\right)$. No significant effect was found for group. Mean comparison between groups did not show significant differences in time 1. For time 3, significant differences between groups were found, $\mathrm{t}(26)=3.64, \mathrm{p}<.001, d=-1.39$. Significant differences were also found in the intragroup comparison for the treatment group, $\mathrm{t}(15)=6.25, \mathrm{p}<.001, d$ $=1.41$, between the two measurements. No significant differences were observed in the non-treatment TAU group between the different time applications. See Table 1 and figure 3 .

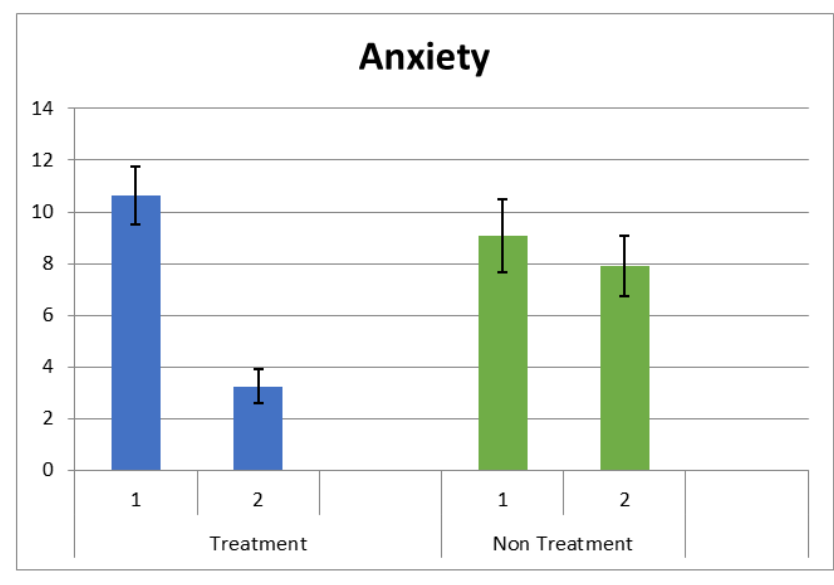

Figure 3. Mean scores and standard error for Anxiety by time and group.

\section{Depression}

Significant effects were found for time $(\mathrm{F}(1,26)=21.77$, $\left.\mathrm{p}<.001, \eta_{P}^{2}=.456\right)$. Results also showed significant interaction effects between time and group. $(\mathrm{F}(1,26)=6.17$, $\left.\mathrm{p}<.05, \eta_{P}^{2}=.192\right)$. No significant effects for group were found. Comparison between groups did not showed significant differences in time 1 , for time 3 not significant differences were found. For the treatment group significant differences were confirmed between the two measurements, $t$ $(15)=5.18, \mathrm{p}<.001, d=1.02)$. No significant differences were found for intragroup comparisons in the TAU group. See Table 1 and figure 4.

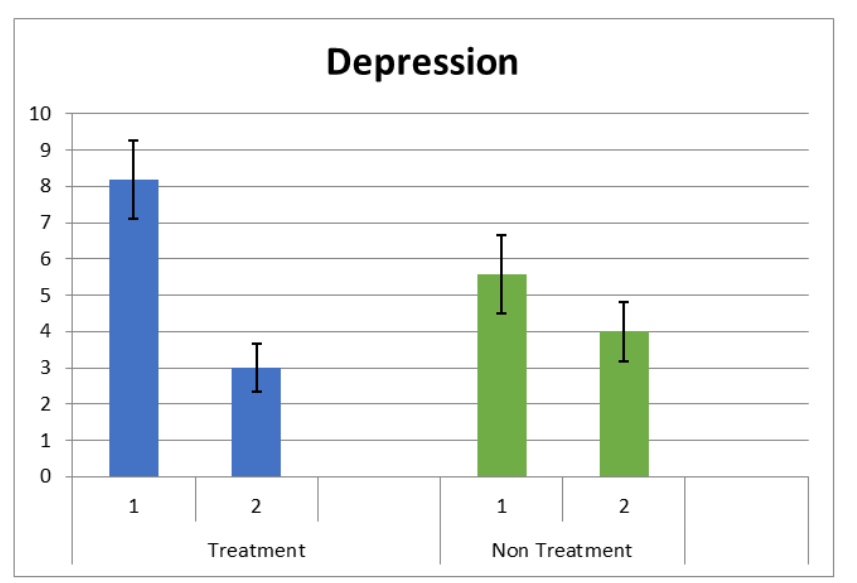

Figure 4. Mean scores and standard error for Depression by time and group.

PCL-5 Core Criteria Provisional PTSD Diagnosis Correlation with CAPS-5 Clinical Diagnosis.

At Time 1 (pre-treatment assessment), 29 out of 32 (90.62\%) of PCL-5 core criteria provisional PTSD diagnosis correlated with the CAPS-5 clinical diagnosis. At time 2 (post-treatment) and Time 3 (follow-up) assessments, 28 out of $28(100 \%)$ of PCL-5 provisional PTSD diagnosis correlated with the CAPS-5 clinical diagnosis.

\section{Discussion}

This longitudinal multicenter randomized controlled trial has three objectives 1 ) to evaluate the effectiveness of the eye 
movement desensitization and reprocessing Protocol for Recent Critical Incidents and Ongoing Traumatic Stress (EMDR-PRECI) in the remission of the Posttraumatic Stress Disorder (PTSD) Diagnosis on female minors who were victims of sexual and/or physical violence and related PTSD diagnosis compared to treatment as usual (TAU), 2) to evaluate the effectiveness of the EMDR-PRECI in the reduction of the PTSD, anxiety, and depression symptoms of the participants compared with TAU, and 3) to expand the knowledge on the existing correlation between the Posttraumatic Stress Disorder Checklist for DSM-5 (PCL-5) core criteria for PTSD provisional diagnosis and the Clinician-Administered PTSD Scale for DSM-5 (CAPS-5) PTSD clinical diagnosis on this population.

Regarding the first objective, results according the DSM-5 diagnostic algorithm, showed that EMDR-PRECI was effective for the remission of the Posttraumatic Stress Disorder (PTSD) diagnosis on female minors who were victims of sexual and/or physical violence. A clear significant decrease was observed in PTSD symptoms, between the first and second score, which also decreased between the second and third measurement, in the treatment group. It is important to mention that differences between second and third measure in the treatment group showed a considerable trend toward significance (.06) confirming the reprocessing of pathogenic memories. It is also clear that scores in the TAU group were maintained practically at the same level along the study. It is relevant to report that $50 \%$ of the EMDR treatment group participants, reported being asymptomatic, and the other $50 \%$ reported a considerable improvement at follow-up assessments.

The evaluation of the effectiveness of the EMDR-PRECI in the reduction of PTSD, anxiety and depression symptoms is showed through the strong effect $(d=7.90)$ resulted in the comparison between the two groups at Time 3 for PSTD. Results also showed the effectiveness of the protocol for the reduction of anxiety and depression. For both variables, the decrease in the treatment group is clear at the posttest, confirmed by the strong effect size $(d=1.41)$ in the mean comparison for anxiety. A similar pattern was observed for depression, which also demonstrated a strong effect size $(d=1.02)$.

In reference to the existing correlation between the PCL-5 core criteria for PTSD provisional diagnosis and the CAPS-5 PTSD clinical diagnosis on this population, results showed a $90.62 \%$ of correlation at Time 1 , and a $100 \%$ correlation at Times 2, and 3 .

Minors and women have suffered interpersonal violence for centuries. Among other manifestations, interpersonal violence includes sexual and/or physical violence, intimate partner violence, family or parental violence, and genderbased violence. Multiple interpersonal traumatic experiences which occur in childhood and adolescence (e.g., sexual and/or physical violence, emotional abuse or neglect), have been defined as "complex trauma" [49-50]. Complex trauma increases the risk of PTSD and also a variety of emotional, behavioral and physical problems, including anxiety, depression, aggression, loss of core capacities for emotional self-regulation and interpersonal relatedness, dissociation, addictive disorders, physical illness, low self- esteem and sexualized behavior. Recent, present or past prolonged adverse experiences lived by these populations requires a specific kind of treatment approach to encompass their whole traumatic stress spectrum, like the one provided with the EMDR-PRECI.

\section{Conclusion}

Sexual violence is one of the most serious forms of interpersonal violence a minor can suffer, because it is always associated with other forms of abuse like emotional and/or physical abuse. Besides PTSD, anxiety and depression symptoms; sexually abused minors can show psychosomatic, obsessive, and phobic symptoms, as well as school failures, insomnia, listlessness, social isolation, low self-esteem and escape from home. The study results indicate that the administration of the EMDR-PRECI could be a feasible, cost-effective, and time-efficient therapy to address PTSD diagnosis, and PTSD, anxiety and depression symptoms in this population.

\section{Limitations and Future Directions}

The small sample size and the follow-up assessment at three months are limitations of this study. We recommend future randomized controlled trial with a larger sample, with follow-up assessment at six months and following the Consolidated Standards of Reporting Trials (CONSORT) 2010 Statement and the Standard Protocol Items Recommendation for Interventional Trials (SPIRIT) 2013 checklist.

\section{Conflict of Interest and Founding}

The authors declare that they have no competing interest.

\section{Acknowledgements}

We want to express our gratitude for their support during this study to María Elena Estevez, Susana Uribe, Yara Brom, and Lynne Levinson.

\section{References}

[1] UNICEF, "Prevention and response to violence against children and adolescents, theory of change", Child Protection Section, New York, 2017b. Retrieved from: <www. UNICEF. org / protection / files UNICEF_VAC_ToC_WEB_271117.pdf $>$

[2] Leander, L., Garnhag P. A., \& Christianson, S. A. (2005). Children exposed to obscene phone calls: what they remember and say., Child Abuse \& Neglect 29 (8), 871-888. http://dx.doi.org/10.1016/j.chiabu.2004.12.012 
[3] Amstadter, A. B., \& Vernon, L. L. (2008) Emotional Reactions During and After Trauma: A Comparison of Trauma Types. Journal of Aggression, Maltreatment \& Trauma. 2008; 16 (4): 391-408.

[4] Nooner, K. B., Linares, L. O., Batinjane, J., Kramer, R. A., Silva, R., Cloitre, M. (2012) Factors Related to Posttraumatic Stress Disorder in Adolescence. Trauma, Violence, \& Abuse. 2012; 13 (3): 153-66.

[5] Collin-Vézina, D., \& Hébert, M. (2005). Comparing dissociation and PTSD in sexually abused school-aged girls. Journal of Nervous and Mental Disease, 193 (1), 47-52.

[6] Cutajar, M. C., Mullen, P. E., Ogloff, J. R. P., Thomas, S. D., Wells, D. L., \& Spataro, J. (2010). Psychopathology in a large cohort of sexually abused children followed up to 43 years. Child Abuse \& Neglect, 34 (11), 813-822. http://dx.doi.org/10.1016/j.chiabu.2010.04.004.

[7] Grassi-Oliveira, R. (2005). Child maltreatment: assessment tools and study of association with posttraumatic stress disorder and general psychiatric symptoms in adults. Unpublished Master's Dissertation, Postgraduate Program in Psychology, Pontifical Catholic University of Rio Grande do Sul, Porto Alegre.

[8] Borges, J., \& Dalbosco Dell'Aglio, D. (2008) Relationships between sexual abuse in childhood, post-traumatic stress disorder (PTSD) and cognitive impairments. Article in Psicologia em Estudo. Maringá, v. 13, n. 2, p. 371-379, abr./jun. 2008.

[9] Mexican National Institute of Statistics and Geography, National survey on the dynamics of relationships in households. Mexico: INEGI, 2016.

[10] OECD (2019). Mexico and sexual abuse. Bulletins 45796. Senate of the Mexican Republic. http: //comunicacion.senado.gob.mx/index.php/informacion/boletin es/45796-mexico-primer-lugar-en-abuso-sexual-infantil.html.

[11] Fontes, L. A., \& Plummer, C. (2010). Cultural issues in disclosures of child sexual abuse. Journal of Child Sexual Abuse, 19, 491-518. http://dx.doi.org/10.1080/10538712.2010.512520

[12] American Psychiatric Association. (2013). Diagnostic and statistical manual of mental disorders $\left(5^{\text {th }} \mathrm{ed}\right.$.). Arlington, VA.

[13] J. Sareen, Posttraumatic stress disorder in adults: impact, comorbidity, risk factors, and treatment (2014). Canadian Journal of Psychiatry, Rev. Canad. Psychiatr. 59 (9), 460-467 https://doi.org/10.1177/070674371405900902

[14] L. A. Brown, A. Jerud, A. Asnaani, J. Petersen, Y. Zang, \& E. B. Foa, (2018). Changes in posttraumatic stress disorder (PTSD) and depressive symptoms over the course of prolonged exposure, J. Consult. Clin. Psychol. 86 (5) 452$463 \mathrm{https}: / /$ doi.org/10.1037/ccp0000292.

[15] Seng, J., Oakley, D., Sampselle, C., Killion, C., GrahamBermann, S., \& Liberzon, I., (2001) Posttraumatic stress disorder and pregnancy complications. Obstet Gynecol 2001; 97: 17-22.

[16] Buss, C., Entringer, S., Lu, M., \& Wadhwa, P., (2011) The Contribution of Maternal Stress to Preterm Birth: Issues and Considerations. Clin Perinatol. 2011 Sep; 38 (3): 351-384.

[17] Chen, Y. R., Hung, K. W., Tsai, J. C., Chu, H., Chung, M. H.,
Chen, S. R., ... Chou, K. R. (2014). Efficacy of eyemovement desensitization and reprocessing for patients with posttraumatic stress disorder: A meta-analysis of randomized controlled trials. PLoS One, 9, 8.

[18] American Psychiatric Association. (2017). Clinical practice guideline for the treatment of posttraumatic stress disorder in adults. Retrieved from http://www.apa.org/ptsdguideline/.

[19] International Society for Traumatic Stress Studies (ISTSS). (2018). Posttraumatic stress disorder prevention and treatment guidelines: Methodology and recommendations. Oakbrook Terrace, USA: ISTSS.

[20] U.S. Department of Veterans Affairs, Department of Defense (2016). VA/DoD clinical practice guideline for the management of posttraumatic stress disorder and acute stress disorder. Washington DC, USA. https://www.healthquality.va.gov/ptsd/ptsd_full.pdf

[21] Shapiro, F. (2018). Eye movements desensitization and reprocessing. Basic principles, protocols, and procedures (Third edition). Guilford Press. N.Y.

[22] World Health Organization. (2013). Guidelines for the management of conditions specifically related to stress. Geneva, Switzerland: Author.

[23] A. M. Khan, S. Dar, R. Ahmed, R. Bachu, M. Adnan, V. P. Kotapati. (2018). Cognitive-behavioral therapy versus eye movement desensitization and reprocessing in patients with post-traumatic stress disorder: systematic review and metaanalysis of randomized clinical trials, Cureus 10 (9) e3250 e3250 https://doi.org/10. 7759/cureus.3250.

[24] Beer, R. (2018). Efficacy of EMDR therapy for children with PTSD: A review of the literature. Journal of EMDR Practice and Research, 12, 4. doi: 10.1891/1933-3196.12.4.177.

[25] Morris-Smith, J., \& Silvestre, M. (2014). EMDR for the next generation: Healing children and families (2nd ed.). United Kingdom: Academic Conferences and Publishing International.

[26] Jarero, I., \& Artigas, L. (2018). AIP-model Based Acute Trauma and Ongoing Traumatic Stress Theoretical Conceptualization. Iberoamerican Journal of Psychotraumatology and Dissociation, 10 (1), 1-10. Retrieved from https://www.revibapst.com/volumen-10-numero-1-20182019

[27] Jarero, I., Artigas, L., \& Luber, M. (2011). The EMDR protocol for recent critical incidents: Application in a disaster mental health continuum of care context. Journal of EMDR Practice and Research, 5 (3), 82-94.

[28] Jarero, I., \& Uribe, S. (2011). The EMDR protocol for recent critical incidents: Brief report of an application in a human massacre situation. Journal of EMDR Practice and Research, $5(4), 156-166$.

[29] Jarero, I., \& Uribe, S. (2012). The EMDR protocol for recent critical incidents: Follow-up Report of an application in a human massacre situation. Journal of EMDR Practice and Research, 6 (2), 50-61.

[30] Jarero, I., Amaya, C., Givaudan, M., \& Miranda, A. (2013). EMDR Individual Protocol for paraprofessional use: A randomized controlled trial with first responders. Journal of EMDR Practice and Research, 7 (2), 55-64. 
[31] Jarero, I., Uribe, S., Artigas, L., \& Givaudan, M. (2015). EMDR protocol for recent critical incidents: A randomized controlled trial in a technological disaster context. Journal of EMDR Practice and Research, 9 (4), 166-173.

[32] Jarero, I., Schnaider, S., \& Givaudan, M. (2019). EMDR Protocol for Recent Critical Incidents and Ongoing Traumatic Stress with First Responders: A Randomized Controlled Trial. Journal of EMDR Practice and Research, 13 (2), 100-110.

[33] Encinas, M., Osorio, A., Jarero, I., \& Givaudan, M. (2019). Randomized Controlled Clinical Trial on the Provision of the EMDR-PRECI to Family Caregivers of Patients with Autism Spectrum Disorder. Psychology and Behavioral Science International Journal, 11 (1), 1-8.

[34] Estrada, B. D., Angulo, B. J., Navarro, M. E., Jarero, I., Sánchez-Armass, O. (2019). PTSD, Immunoglobulins, and Cortisol Changes after the Provision of the EMDR-PRECI to Females Patients with Cancer-Related PTSD Diagnosis. American Journal of Applied Psychology, 8 (3), 64-71.

[35] Consolidated Standards of Reporting Trials (CONSORT) 2010 Statement. BMJ 2010; 340: c869 doi: 10.1136/bmj.c869

[36] Standard Protocol Items Recommendation for Interventional Trials (SPIRIT) 2013 Checklist. Retrieved at http://www.spirit-statement.org/wpcontent/uploads/2013/01/SPIRIT-Checklist-download8Jan13.pdf

[37] Weathers, F. W., Blake, D. D., Schnurr, P. P., Kaloupek, D. G., Marx, B. P., \& Keane, T. M. (2015). The ClinicianAdministered PTSD Scale for DSM-5 (CAPS-5) - Past Month [Measurement instrument]. Available from https://www.ptsd.va.gov/

[38] Weathers, F. W., Litz, B. T., Keane, T. M., Palmieri, P. A., Marx, B. P., \& Schnurr, P. P. (2013). The PTSD Checklist for DSM-5 (PCL-5). Scale available from the National Center for PTSD at www.ptsd.va.gov

[39] Bovin, M. J., Marx, B. P., Weathers, F. W., Gallagher, M. W., Rodriguez, P., et al. (2016) Psychometric properties of the PTSD checklist for diagnostic and statistical manual of mental disorders- Firth edition (PCL-5) in veterans. Psychol Assess 28 (11): 1379-1391.
[40] American Psychiatric Association. (2000). Diagnostic and statistical manual of mental disorders (4th ed. Text Revised). Arlington, VA.

[41] Weathers, F. W., Blake, D. D., Schnurr, P. P., Kaloupek, D. G., Marx, B. P., Keane, T. M., 2013a. Clinician-administered PTSD Scale for DSM-5. National Center for Posttraumatic Stress Disorder, Boston.

[42] Zigmond, A. S., \& Snaith, R. P. (1983). The Hospital Anxiety and Depression Scale. Acta Psychiatrica Scandinavica, 67, 361-370.

[43] Ying Lin C, \& Pakpour AH (2017) Using Hospital Anxiety and Depression Scale (HADS) on patients with epilepsy: Confirmatory factor analysis and Rasch models. Seizure (45): 42-46.

[44] Hurley, E. C. (2018). Effective Treatment of Veterans With PTSD: Comparison Between Intensive Daily and Weekly EMDR Approaches. Front. Psychol. 9: 1458. doi: 10.3389/fpsyg.2018.01458.

[45] Bongaerts, H., Van Minnen, A., \& de Jongh, A. (2017). Intensive EMDR to treat patients with complex posttraumatic stress disorder: A case series. Journal of EMDR Practice and Research, 11 (2), 84-95.

[46] Gutner, C. A., Suvak, M. K., Sloan, D. M., \& Resick, P. A. (2016). Does timing matter? Examining the impact of session timing on outcome. Journal of Consulting and Clinical Psychology, 84, 1108-1115.

[47] Holmes et al. (2011). Imagery about suicide in depression" Flash-forwards"? Journal of Behavior Therapy \& Experimental Psychiatry, 38, 423-434.

[48] Logie, R., \& de Jongh, A. (2014). The "flashforward" procedure: Confronting thecatastrophe. Journal of EMDR Practice and Research, 8, 25-32.

[49] Kezelman, C., \& Stavropoulos, P. (2012). The Last Frontier:' Practice Guidelines for the Treatment of Complex Trauma and Trauma-Informed Care and Service Delivery. Kirribilli: Adults Surviving Child Abuse.

[50] Cook, A., Spinazzola, J., Ford, J., Lanktree, C., Blaustein, M., Cloitre, M., et al. (2017). Complex trauma in children and adolescents. Psychiatr. Ann. 35, 390-398. 